\title{
SBML and MathML support for the stochastic reaction-diffusion simulator STEPS
}

\author{
lain Hepburn ${ }^{1,2^{*}}$, Weiliang Chen ${ }^{1}$, Erik De Schutter ${ }^{1,2}$ \\ From Twentieth Annual Computational Neuroscience Meeting: CNS*2011 \\ Stockholm, Sweden. 23-28 July 2011
}

STEPS is a stochastic signaling pathway simulator based on the "spatial Gillespie" method for reaction-diffusion simulation, within a Python interface [1]. STEPS supports tetrahedral meshes, which allow for complex boundary representation absent from simulators based on cubic voxels. A powerful addition to STEPS, the membrane potential calculation, is under development. The potential of nodes throughout the tetrahedral mesh are simulated, which allows for the inclusion of voltage-gated membrane channels with molecular channel currents within the simulation.

STEPS supports spatial and non-spatial simulations, and future versions will even allow for a combination of spatial and well-mixed compartments in one simulation. The Systems Biology Markup Language (SBML) is a widely used format for representing models of networks of biochemical interactions. The neuronal signaling pathways that STEPS is designed to simulate in a wellmixed context fall under this umbrella.

While it was found that the Python interfaces to STEPS and libSBML makes it an ease to support most components of SBML, in some models there may be hurdles that cannot be overcome in a STEPS context and also any other stochastic simulation context. Stochastic simulation approaches, including the SSA that STEPS is based on, assumes that each reaction is the most fundamental with no intermediate, non-simulated steps. Therefore the expected form of the mathematics for the reaction rate is easily definable, but may differ from the explicit mathematics specified in some SBML files. If STEPS were to ignore alternate reaction kinetic law mathematics then any model that included such would be represented incorrectly. An example is a catalyst reaction that may often be described as a single reaction whose kinetic mathematics is influenced by the catalyst, which should

\footnotetext{
* Correspondence: ihepburn@oist.jp

${ }^{1}$ Computational Neuroscience Unit, OIST, Okinawa, 904-0411 Japan

Full list of author information is available at the end of the article
}

typically be broken down into three or more separate reactions in the stochastic description.

STEPS performs a comparison of the reaction kinetic law mathematics to the form that is expected, which means that a model will never be simulated incorrectly. This is achieved by converting the MathML components in SBML, including reaction kinetic law mathematics, to Python objects with no limit on depth. MathML support in the Python interface then makes it easy to support other features of SBML that are based on MathML, which are numerous. This is because the MathML Python objects are available during simulation and may reference any variable in the model including those whose value is not constant during simulation. This extends SBML support in STEPS to many components that other stochastic simulators cannot support because they ignore MathML, including important features such as Initial Assignments, Assignment Rules, Rate Rules and Events.

\section{Author details}

'Computational Neuroscience Unit, OIST, Okinawa, 904-0411 Japan.

${ }^{2}$ Theoretical Neurobiology, University of Antwerp, B-2610 Antwerpen, Belgium.

Published: 18 July 2011

\section{References}

1. Wils S, De Schutter E: STEPS: Modeling and simulating complex reactiondiffusion systems with Python. Front. Neuroinf 2009, 3:15.

2. Hucka M, Finney A, Sauro HM, Bolouri H, Doyle JC, Kitano H, Arkin AP, Bornstein BJ, Bray D, Cornish-Bowden A, Cuellar AA, Dronov S, Gilles ED, Ginkel M, Gor V, Goryanin II, Hedley WJ, Hodgman TC, Hofmeyr J-H, Hunter PJ, Juty NS, Kasberger JL, Kremling A, Kummer U, Le Novère N, Loew LM, Lucio D, Mendes P, Minch E, Mjolsness ED, Nakayama Y, Nelson MR, Nielsen PF, Sakurada T, Schaff JC, Shapiro BE, Shimizu TS, Spence HD, Stelling J, Takahashi K, Tomita M, Wagner J, Wang J: The Systems Biology Markup Language (SBML): A Medium for Representation and Exchange of Biochemical Network Models. Bioinformatics 2003, 9(4):524-531.

doi:10.1186/1471-2202-12-S1-P57

Cite this article as: Hepburn et al: SBML and MathML support for the stochastic reaction-diffusion simulator STEPS. BMC Neuroscience 201112 (Suppl 1):P57.
C Biomed Central

() 2011 Hepburn et al; licensee BioMed Central Ltd. This is an open access article distributed under the terms of the Creative Commons Attribution License (http://creativecommons.org/licenses/by/2.0), which permits unrestricted use, distribution, and reproduction in any medium, provided the original work is properly cited. 